\title{
Ageing Populations in Post-Industrial Democracies: Comparative Studies of Policies and Politics
}

\author{
Edited by Pieter Vanhuysse and Achim Goerres \\ New York: Routledge/ECPR 2012 \\ ISBN: 978-0415603829 \\ Hardcover, \$125.00, 296 pp.
}

\author{
Reviewed by Michel Grignon \\ Departments of Economics and of Health, Aging, and Society, McMaster University
}

and

\author{
Byron G. Spencer \\ Department of Economics, McMaster University \\ spencer@mcmaster.ca
}

While the consequences of population ageing in developed and democratic nations have been considered by scholars from many disciplines, the editors note that "political science as a discipline has lagged behind in developing an integrated body of knowledge to answer the question of which generations get what, when, and how," and state that this volume "aims to be a building block for such a body of research" (p. 1). The two main research questions explored throughout the volume are: (a) Can it be said that generational warfare has replaced class warfare in industrialized countries? and (b) Are ageing democratic societies driven by the growing electoral clout of the elderly (the elderly power hypothesis) or rather by cost-containment policies targeted toward the elderly (so-called leakage hypothesis)?

The volume brings together a collection of eleven studies from twelve scholars in six countries, and provides a mix of quantitative and qualitative studies, each with an "explicitly comparative-political lens" (p. 9), intended to explore how political systems function as major generational changes take place in the context of fiscal restraint. The studies are diverse in the range of approaches that are used (some following a rational public choice approach, other relying more on path dependence and institutionalist perspectives) and, we find, insightful in the conclusions that are reached.

The editors do a nice job of "mapping the field" in their introductory chapter, but an important quibble relates to their (widely shared) use of 65 and older as the marker of old when most of their comparisons extend extend over six decades; given that they emphasize the observed increases in life expectancy (including healthy life expectancy), it seems odd to continue to fixate on age 65. Indeed, the worsening of the dependency ratio would be largely offset if the concept of old were adjusted to reflect such gains; that point is noted (p. 8) but then ignored. In any event, population projections do not play a major role in the chapters that follow. There are a few unfortunate editorial lapses: one is the occasional careless use of numbers, with a notable example appearing in Table 1.1, bottom panel, which reports, "Elderly care costs as a share of GDP" in 1980 and 2005. For the OECD-30 it is reported as 0.58 in 2005, meaning that a preposterous 58 per cent of GDP was used in this way, even though the text interprets it as " 0.58 per cent of GDP" (p. 8); furthermore, there is no description of the concept that is measured, and the source note is not sufficiently informative to enable readers to investigate. The long-run decline in the labour force participation rate of 
older male workers is noted, together with the concurrent increase in the female rate, but the fact that the overall (both sex) rate in that age group has increased is not mentioned.

Such problems aside, this volume has much to offer. Seán Hanley uses both crisp and fuzzy set variants of a qualitative comparative analysis technique, with seven variables that he derives to gain some understanding of the success of pensioners' parties in 31 western and eastern European countries; he describes his results as "suggestive rather than conclusive" (p. 45) and finds that no combination of variables seems to provide a satisfactory explanation of the (marginal and rare) successes of these pensioners' parties. The main conclusion is that the causes of success of such parties seem to differ widely between the western European countries and former communist ones.

Jennifer Dabbs Sciubba focuses on Germany, Italy, and Japan, the three "oldest" democracies, to study differences in labour policy, identified as "an understudied arena of generational politics" (p. 55). She finds that the «connections between population ageing and political power are complex» and that while some parties focus on the interests of particular age groups (often the young, contrary to what one might expect), the more general concern is to "combat the negative ramifications of population ageing" (p. 73), and that means making better use of available resources by keeping the young employed and the old in employment. That casts doubt, as she notes, on the "elderly power hypothesis" (p. 73). We find that the study is not clear enough on the criteria used to determine whether a policy favours or works against a given age group (e.g., policies to help the young to enter the labour force are labelled "pro-young" while policies to encourage the elderly to stay in the labour force are labelled "anti-elderly").

Martin Hering, in a nicely nuanced study of reforms in Germany and the UK, concludes that "even though retirement age changes [read increases] are extremely unpopular, they are politically feasible" (p. 100), but that when changes are made, the implications for equity across generations are not carefully considered and do not seem to be the main motivation behind the reform. The chapter shows that, faced with two constraints (fiscal constraint and poverty rate among the elderly), policymakers had no choice but to increase the retirement age. The chapter also shows that despite very different institutions and political cultures, both countries made similar choices and used similar blame-avoiding tools (such as commissions and grand coalitions). This highly convincing account of reforms in these two countries would seem to apply elsewhere as well, the Sarkozy reform in France being one example. However, we note that even though an increase in the retirement age may be the least preferred option in surveys, it need not be highly unpopular: in one-on-one comparisons, the young prefer cutting benefits but would rank raising the eligibility age before an increase in contribution rates, while the elderly would prefer to increase contributions but would rank the age reform above benefit cuts.

Next, Mehmet Aysan asks "whether the pressures of globalization and demographics bring convergence across welfare states, or whether welfare states follow their traditional welfare regime paths in pension reforms" (p. 106). He uses cluster analysis applied to fourteen indicators of pension policies in nineteen OECD countries, and concludes that convergence is much constrained by the institutional characteristics of welfare regimes. We find that not all indicators are well defined, and that the source notes do not make it possible to determine precisely what they are; by way of example, what is included in the numerator of "old age expenditure as percentage of GDP"? And what is "government spending on old age"?

Markus Tepe and Pieter Vanhuysse make effective use of event history analysis with data from eighteen OECD welfare states over a 19-year period ending in 1999, to test alternative theoretical perspectives relating to the "motive or the ability to delay generosity in [pension] cutbacks" (p. 127). They are concerned with cutbacks that took place within three years, and distinguish between medium (8 per cent) and large (12 per cent) cutbacks. The relatively short (three-year) time horizon means that it is difficult for politicians to avoid blame. They find, contrary to expectations, that "neither the number of years left in the current electoral cycle nor the degree of institutional rigidity in the political system turned out to have a significant effect on timing" (p. 139), but that right-wing parties implement cutbacks more quickly; that medium-size cuts are twice as common as large ones; and, interestingly, that "crises deriving from mounting unemployment and population ageing may lead governments to accelerate medium-sized generosity cutbacks while delaying large-sized ones” (p. 139). A concern is that event history (duration) analysis relies on the assumption that all countries will at some point implement a major or medium-sized cutback. Empirical data presented in the chapter, however, show that this 
is not the case; as a result, their estimated hazard might be biased. A split-population duration model might be a better approach. In any event, research following this approach opens a new path for our understanding of how governments adjust to population ageing in times of fiscal restraint.

How population ageing affects the generosity of public pension plans is the concern of Juan Fernández's innovative and thoughtful study. "Generosity" is appropriately measured by two synthetic replacement rates, one being the fraction of salary that would be replaced in the first year of retirement for an average production worker who contributed to the plan for 40 years (the "standard" pension), and the other being the fraction of the same average salary that would be replaced for a person with no contribution history (the "minimum" pension). The most interesting major finding is that the beneficiaries approach (elderly power hypothesis) has the most explanatory power, and is positively associated with standard pension replacement rates and negatively associated with minimum pension replacement rates. The analysis is based on data from twenty-one OECD countries over a 23-year period ending in 2002, with demographic (share of elderly population), political (left cabinet portfolio, right cabinet portfolio, constitutional structure, women in parliament), and economic (GDP per capita, net trade, public deficit, GDP growth, and inflation) explanatory variables. The use of "net trade," defined as imports less exports as a fraction of GDP, seems a strange choice for a measure of "globalization;" that is because both imports and exports could be large relative to GDP, indicating an economy that is active in global markets, even though the difference is zero; the sum of the two relative to GDP would be a better measure. Here again, a concern is that not all variables are carefully defined; "Swank's dataset (2007)" is mentioned (p. 158), but not included in the references. Also, we are concerned about using only the estimated coefficients relating to age distribution to infer the quantitative importance of ageing in the extreme cases (Sweden and Canada), without taking account of the other major differences in those countries that is reflected in the estimation.

The chapter by Achim Goerres and Markus Tepe focuses on what conditions the attitudes of young people (under age 45) towards the public provision of family services. They use survey data from 21 OECD countries to ask how expectations are conditioned by the existing family policy regime and, given the regime, how that relates to family solidarity. Four regime types are distinguished, ranging from those that provide strong support for dual-earner families to those that provide little such support. Family solidarity is measured by the frequency of parental visits, agreement with the view that children should look after their parents, and whether parents would be asked for assistance in case of illness. The explanatory power of their models is low, as would be expected with survey data, but the analysis is careful and thoughtful. A suggestion is that multi-level modelling might be used when country-level variables are introduced.

Jonas Edlund and Stefan Svallfors take as their starting point prior evidence of "substantial convergence across countries in their citizens' views about government responsibilities" (p. 206), and ask whether that might be the result of attitudinal changes across cohorts or classes (which they interpret to mean occupation). Their analysis, which focuses on attitudes toward income redistribution and unemployment policies in the UK and the US, is based on data drawn from the International Social Survey Programme for 1996 and 2006. Their approach is simple but telling, and they conclude that the observed convergence, at least as it relates to these two matters, cannot be explained by cohort replacement, but that "changing class patterns in Britain account for some of the convergence" (p. 218). They conclude also that their findings "speak against any scenario of an emerging 'generational war"' (p. 218).

Andrej Kokkonen explores the impact of family policies on the likelihood of women living in unions as a possible causal path affecting fertility. Using multilevel modelling with 2003 and 2006 rounds of the European Social Survey, he finds strong support for his hypothesis that while working women are less likely than nonworking women to live in unions, they are more likely to live in unions if the countries in which they live have generous dual-earner policies (i.e., paid parental leave and childcare subsidies). He argues convincingly that studies of the determinants of fertility that are restricted to married women may lead to spurious findings.

Robert Hudson's "epilogue" combines a masterful review of the distinctive development of American welfare policies with a comparative perspective that draws on the previous chapters as well as the broader literature.

All in all, this is a rewarding book, and one that has significantly enhanced our understanding of how decision-making interacts with the environment in which it takes place. 